\title{
Derivation of a countrywide river network based on Airborne Laser Scanning DEMs - results of a pilot study
}

\author{
G. Mandlburger $^{\mathrm{a}}$, M. Vetter $^{\mathrm{b}}$, M. Milenkovic ${ }^{\mathrm{a}}$ and N. Pfeifer ${ }^{\mathrm{a}}$ \\ ${ }^{a}$ Vienna University of Technology, Institute of Photogrammetry and Remote Sensing, \\ Gusshausstraße 27-29, A-1040 Vienna, Austria \\ ${ }^{\mathrm{b}}$ Vienna University of Technology, Centre for Water Resource Systems, A-1040 Vienna, Austria \\ Email: gm@ipf.tuwien.ac.at
}

\begin{abstract}
In this paper we present the results of a pilot study carried out for the Austrian Federal Ministry of Agriculture, Forestry, Environment and Water Management. The main goal is to provide a robust method for the extraction of the countrywide river network based on $1 \mathrm{~m}$ Digital Terrain and Surface Models (DTM/DSM) derived from Airborne Laser Scanning (ALS) for rivers with a catchment area less than $100 \mathrm{~km}^{2}$. The basic motivation is to update and improve the geometric quality of the existing river network which was formerly mapped from stereo images $(1: 30,000)$. Furthermore, the final product is expected in compliance with a predefined GIS data model implementing the requirements of the European Water Framework Directive (WFD), i.e., a topologically consistent route model with additional attributes per river section (bridges, bed slope, stream ordering number). As far as possible, the entire workflow should consider the available IT infrastructure of the contracting authority. Thus, the suitability of both commercial software and open source products (ArcGIS, GRASS GIS, SAGA) was investigated.
\end{abstract}

Due to the sheer amount and the heterogeneity of the ALS data the straight forward application of standard channel network extraction routines based on flow accumulation was not possible. Consequently, a hierarchical 4-step approach was chosen: (i) delineation of catchment areas, (ii) DTM preprocessing, (iii) derivation of the river network per catchment, and (iv) post processing. In a first step, the main watersheds $\left(10-20 \mathrm{~km}^{2}\right)$ are delineated based on a coarse $50 \mathrm{~m}$ DTM grid. On catchment level, the $1 \mathrm{~m}$-DTM is adaptively smoothed, the sinks are removed, and an additional openness map is created. Based on the enhanced DTM the channel network is derived using standard flow accumulation strategies in two passes, first, using a reduced $5 \mathrm{~m}$ resolution and, finally, based on the original $1 \mathrm{~m}$ grid. Artificial stream segments due to micro-morphology structures in the ALS DTM are automatically removed based on the openness layer. In a postprocessing step, the individual catchment networks are merged and thinned, homogeneous river reaches are identified and additional features are derived per reach.

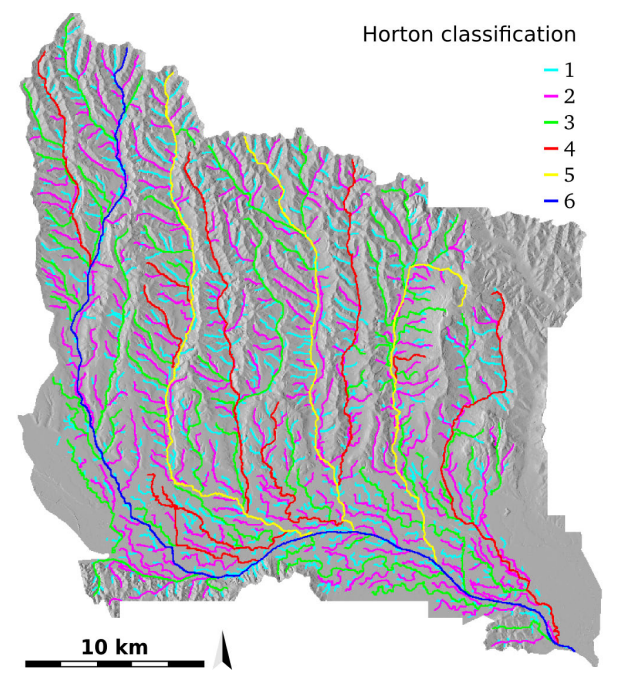

Figure 1: River network derived for a $845 \mathrm{~km}^{2}$ catchment (study area: Styria, Grabenland) based on a $1 \mathrm{~m}$ ALS DTM

The entire workflow was implemented in each of the investigated GIS and was successfully tested at three selected study areas across Austria, each varying in size (80-850 $\left.\mathrm{km}^{2}\right)$ and terrain type (mountainous, undulating, predominantly flat). The results of the pilot study (c.f. Figure 1) demonstrate the high potential for deriving countrywide river networks based on dense ALS datasets in an efficient, accurate, and reliable way with only limited user interaction.

Keywords: ALS, Catchment delineation, River network, Countrywide DEM, GIS 


\section{INTRODUCTION}

Within the last decade, the availability of countrywide Airborne Laser Scanning (ALS) datasets featuring high resolution $\left(>1\right.$ point $\left./ \mathrm{m}^{2}\right)$ and height accuracy $(<15 \mathrm{~cm})$ has steadily increased. In Europe, many countries are currently working on finishing their national ALS campaigns (e.g. AT, DE) whereas others are already updating their initial datasets with higher densities (e.g. NL). These datasets provide a valuable source for many fields of application like city and infrastructure planning, disaster management and prevention, environmental monitoring, ecology, forestry, geomorphology, hydrology and the like.

In Austria, the countrywide ALS data capturing is conducted independently by the surveying departments of the nine federal states which lead to a heterogenous dataset with varying point densities (120 points $\left./ \mathrm{m}^{2}\right)$ and height accuracies $(10-20 \mathrm{~cm}$ ) and, consequently, to terrain models with varying properties. Currently, ca. $70 \%$ of the entire land area of $83,879 \mathrm{~km}^{2}$ is covered by ALS with the remaining parts to be completed within the next two years (c.f. Figure 2). The availability of countrywide ALS data plays an important role for fulfilling legal requirements within Austria and in the context of the European Union (EU). Currently, there are ongoing activities to standardize the data exchange on a transnational level and respective guidelines for basic datasets have already been implemented (INSPIRE, EU (2007)). In particular w.r.t. to hydrology, the European Water Framework Directive (WFD, EU (2000)) obligates the member states to document their fresh water resources.

An accurate and up-to-date countrywide river network is one of the basic datasets which is required by the WFD. A countrywide $1 \mathrm{~m}$ DTM grid of Austria would consist of 580,000 x 294,000 grid posts which is far more than can be processed in a single job using off-the-shelf GIS software. Against this background, the Institute of Photogrammetry and Remote Sensing, TU Vienna, was commissioned to conduct a pilot study for the derivation of a countrywide river network based on high resolution Digital Terrain Models (DTM) and Digital Surface Models (DSM) derived from ALS.

\subsection{Objectives}

The existing countrywide hydrological datasets (river network, catchment areas and lakes) do not meet today's requirements concerning accuracy and topological correctness. The main objective of the pilot study is, therefore, to improve the geometric quality and to derive a topologically correct river network in compliance with a predefined data model implementing the requirements of the INSPIRE guidelines. The main points of the data model are: (i) relational geometry model composed of oriented edges and nodes where each node represents the confluence of two rivers and the stream section between two nodes constitutes a simple, non self-intersecting, oriented polyline with a unique ID, (ii) adjacent stream sections are linked allowing the derivation of an entire flow route from the source to the outlet, (iii) the network represents the river axes in 2D (i.e. no heights), and (iv) for each stream section, additional parameters like mean slope and Horton-Strahler stream ordering number must be provided as metadata.

The planimetric accuracy of the derived river axes depends on the quality of the source data and the terrain properties, thus, has a number of influences which may be hard to separate. However, a generalization of subsequent vertices w.r.t. a maximum deviation of $1 \mathrm{~m}$ is desired. The main result of the pilot study is a documented workflow, so that the derivation of the countrywide river network can be carried out by the respective experts of the contracting authority. For that reason, the available IT infrastructure has to be considered and as far as possible standard GIS software should be used. The following tools were employed in the study: ArcGIS (ESRI, 2011), GRASS GIS (GRASS Development Team, 2011), SAGA (SAGA, 2011) and the scientific laser scanning software OPALS (Mandlburger et al., 2009).

\subsection{Study area and datasets}

Three test sites covering different terrain types were provided in order to set up and validate the workflow. The first dataset (Carinthia, Fragant) is located in the high mountain Alpine region of Austria featuring predominantly steep slopes. The topography of the second dataset (Upper Austria, Hausruck) situated at the foothills of the Alps is dominated by rolling hills and flat terrain with the occasional occurrence of meandering rivers. The largest dataset (Styria, Grabenland) is part of the Pannonian basin and, therefore, predominantly flat. It features a series of parallel North-to-South rills separated by terrace-like hills. The location and topography of the study areas are shown in Figure 2 whereas additional characteristic 
Table 1: Characteristic parameters of the study areas

\begin{tabular}{llrccrrl}
\hline Federal state & Catchment & Area & Longitude & Latitude & $\mathrm{h}_{\min }$ & $\mathrm{h}_{\max }$ & Surface type \\
\hline Carinthia & Fragant & $84 \mathrm{~km}^{2}$ & $12^{\circ} 59^{\prime}-13^{\circ} 07^{\prime}$ & $46^{\circ} 56^{\prime}-47^{\circ} 03^{\prime}$ & $703 \mathrm{~m}$ & $3122 \mathrm{~m}$ & mountainous \\
Upper Austria & Hausruck & $175 \mathrm{~km}^{2}$ & $13^{\circ} 32^{\prime}-13^{\circ} 42^{\prime}$ & $48^{\circ} 21^{\prime}-48^{\circ} 29^{\prime}$ & $322 \mathrm{~m}$ & $791 \mathrm{~m}$ & hilly \\
Styria & Grabenland & $845 \mathrm{~km}^{2}$ & $15^{\circ} 36^{\prime}-16^{\circ} 03^{\prime}$ & $46^{\circ} 39^{\prime}-47^{\circ} 00^{\prime}$ & $196 \mathrm{~m}$ & $608 \mathrm{~m}$ & undulating \\
\hline
\end{tabular}

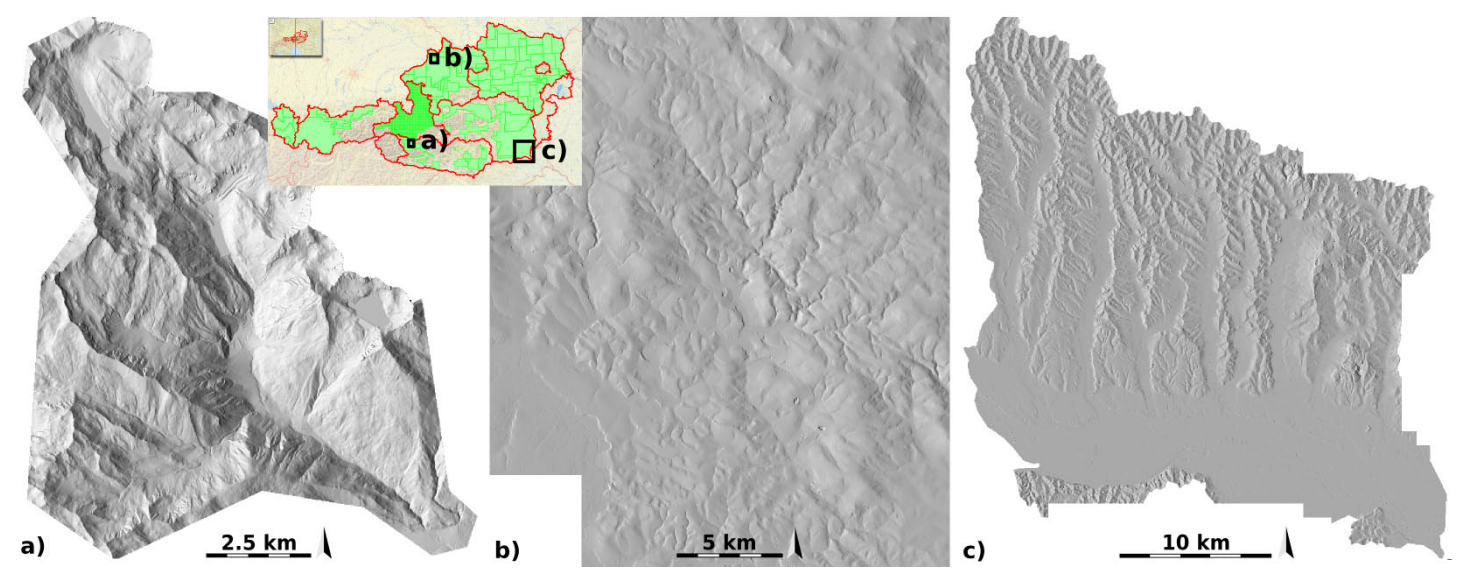

Figure 2: Study areas: (top) current ALS coverage in Austria (July 2011), locations of study areas marked with black rectangles; (a) Carinthia, Fragant, $84 \mathrm{~km}^{2}$; (b) Upper Austria, Hausruck, $175 \mathrm{~km}^{2}$; (c) Styria, Grabenland, $845 \mathrm{~km}^{2}$

parameters are listed in Table 1.

The following datasets were provided by the contracting authority and serve as input data source for all subsequent processing steps: (i) DTM, (ii) DSM, both as regular $1 \mathrm{~m}$ grids, (iii) Digital Orthophotos as geo-referenced $25-50 \mathrm{~cm}$ RGB raster images, and (iv) existing hydrological datasets: river network, catchment areas and a stagnant water layer (lakes, ponds).

\section{METHOD}

In this section the overall workflow to derive the river network is described in detail.

\subsection{Delineation of catchment areas}

In order to be able to derive the river network on a countrywide level based on high resolution ALS DTMs using standard GIS software tools, a subdivision of the entire area into catchments of manageable size $\left(10-20 \mathrm{~km}^{2}\right)$ is necessary. For watershed delineation (Freeman, 1991) a coarse outline is sufficient. Thus, the catchment delineation is carried out based on a $50 \mathrm{~m}$ grid which was downsampled from the original $1 \mathrm{~m}$ grid using a low pass filter. Respective watershed delineation tools are available in all of the employed GIS packages. The catchment polygons have additionally been extended by a $250 \mathrm{~m}$ buffer to avoid underestimation of the effective area. All subsequent processing steps were performed on catchment level and only the final results were blended to a single river network.

\subsection{DTM preprocessing}

Visual inspection: As a precondition for a successful extraction of the river network the DTM has to describe the water surface and, thus, must not contain bridges or other artifacts, as they would act as barriers influencing the actual water flow. Since bridges and filtering artifacts could be identified in some of the DTMs, a preliminary visual inspection and interactive editing is advised (c.f. Section 3).

Smoothing: Since the ALS DTM is to a certain degree affected by measurement noise adaptive smoothing 

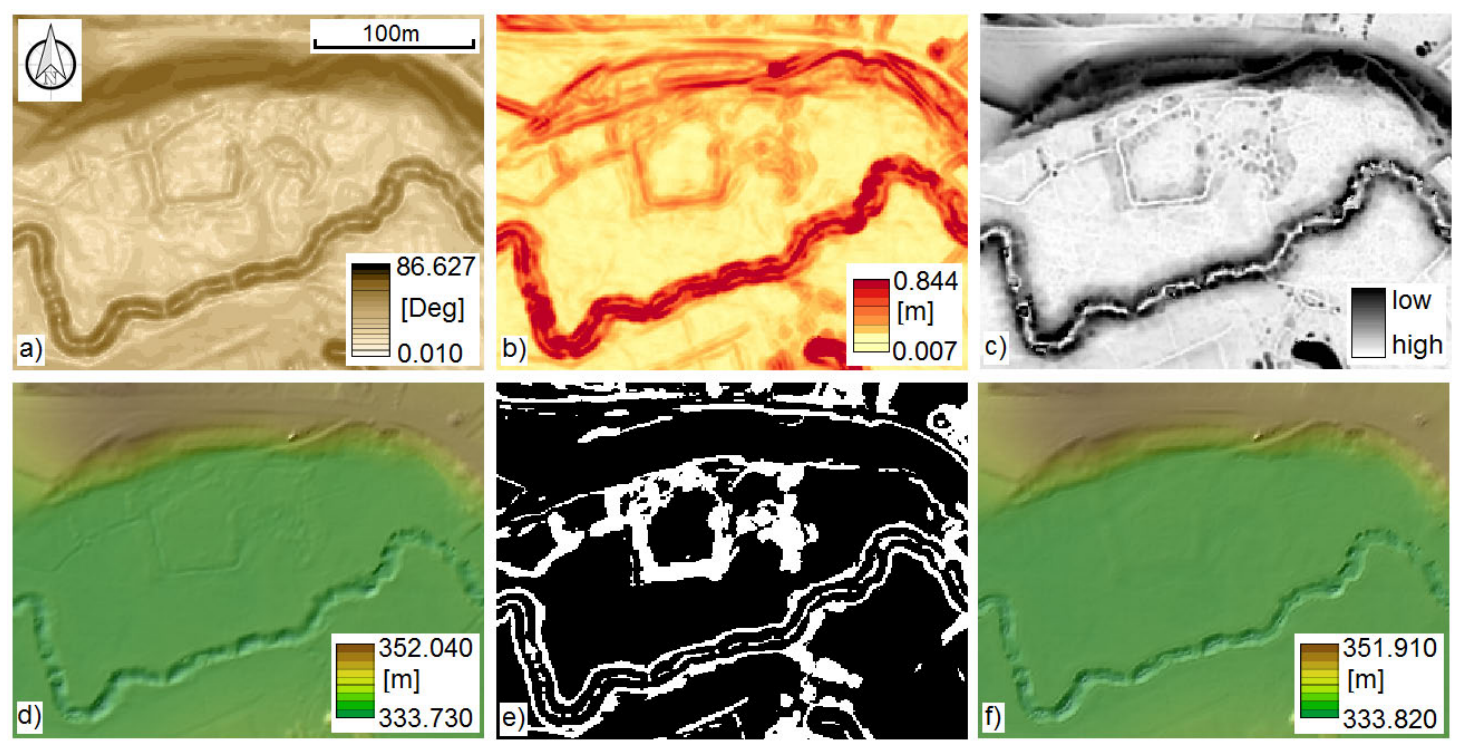

Figure 3: DTM preprocessing, Data: Upper Austria, Hausruck (Lon/Lat: 13³5’38” / 48²3'42”); (a) maximum slope, (b) roughness, (c) negative openness, (d) original $1 \mathrm{~m}$ DTM, (e) smoothing mask (white $=$ smoothing area, black=no smoothing) and (f) adaptively smoothed DTM

is performed to reduce artificial stream segments. First, for each grid point of the original $1 \mathrm{~m}$ DTM the trend is estimated based on the 25 neighboring points (tilted plane regression). As a result the local surface gradient and a roughness measure (i.e. the standard deviation of the plane fit) are obtained additionally to the smoothed heights for each grid post. In a subsequent step the height of either the original or smoothed DTM grid is chosen based on a condition considering the local slope and roughness. The main idea is that smoothing is only required in case the area is both flat and rough (c.f. Figure 3).

Removal of sinks: By applying adaptive smoothing many artificial sinks are filtered. The remaining sinks have to be removed as this is a prerequisite for performing flow accumulation analysis. Sink removal is described in the literature (Jenson and Domingue, 1988) and is implemented in standard GIS software.

Openness: Besides the DTM as primary input, additional surface properties (slope, curvature, openness) can be integrated in the channel network detection process (Sofia et al., 2011). In our study we chose a layer describing the dispersion of negative openness based on a radial distance of $5 \mathrm{~m}$ to remove artificial streams. Openness is an angular measure denoting the aperture angle of a cone centered at a grid point and constrained by the neighboring elevations within a specified radial distance (Yokoyama et al., 2002). It is basically a convexity/concavity measure calculated from local terrain profiles in eight compass directions. A distinction is made between positive and negative openness (viewshed above and below the terrain). The dispersion map describing the local openness variation is calculated as the standard deviation within a $5 \times 5$ kernel.

Resampling: In a final preprocessing step the enhanced $1 \mathrm{~m}$ DTM is resampled to $5 \mathrm{~m}$ resolution using a low pass filter. The enhanced $1 \mathrm{~m}$ and $5 \mathrm{~m}$ DTMs are the basis for all subsequent processing steps.

\subsection{Derivation of river network}

Flow accumulation: The detection of river networks has been well studied in literature and the respective algorithms are available in standard GIS software (Arge et al., 2003). The process mainly relies on a flow accumulation map which, in this study, was derived using a Multiple Flow Directions (MFD) algorithm.

Channel detection: The resulting flow accumulation raster map stores in each pixel a measure equivalent to the overall amount of water received from the upstream cells and, thus, provides the basis for the classification of river pixels by applying a threshold (i.e. minimum required catchment area). The GIS tools (Jasiewicz and Metz, 2011) also provide a classification of all river pixels into separate stream 
segments and by connecting adjacent segments the entire stream network is constructed.

Removal of artificial stream segments: Due to micro-structures available in the ALS DTM artificial stream segments are contained in the raw channel network. To a high degree these false segments can be eliminated automatically based on the openness information. Rivers are concave terrain features following the typical pattern of left foreland, left bank, river, right bank, right foreland. This is reflected in the openness dispersion map as the bank features lower negative openness than the river and the foreland (c.f. Figure 3) and, thus, the dispersion is high for real rivers and low for artificial rivers in open terrain. Consequently, the openness dispersion is an adequate measure to distinguish between real and artificial rivers. In particular, the dispersion of negative openness is extracted for all pixels of a single stream segment and the segment is only confirmed as a real river segment if the mean dispersion is above a threshold.

Refinement of geometry: The above processing steps are first carried out based on the $5 \mathrm{~m}$ DTM grid. In steep terrain, the reduced resolution might be sufficient but in flat areas many details are lost especially for small meandering creeks. To tap the full potential of the ALS data the $1 \mathrm{~m} \mathrm{DTM}$ is extracted within a buffer of $100 \mathrm{~m}$ around the detected rivers and the channel network derivation is repeated. That way, the full $1 \mathrm{~m}$ resolution can be used and, still, the memory consumption and processing times are moderate.

Vectorization and ordering: In a final step on catchment level, the clear river network which, up to now, is available in the raster domain is vectorized and by analyzing the network topology the Horton-Strahler stream ordering numbers are assigned. For all this, standard GIS functionality is available.

\subsection{Postprocessing}

Merging of catchment river networks: After deriving a concise river vector network for each catchment individually, the distinct subnetworks need to be merged to an overall product. Standard GIS packages provide tools for patching multiple vector layers (e.g., GRASS: r.patch). The patching process, hereby, benefits from the prior subdivision into catchment areas as a single watershed features only one outlet, thus, reducing potential ambiguities.

Thinning of final channels: The resulting channel vectors are still affected by high frequent noise which is smoothed by applying a Douglas-Peucker thinning algorithm (Douglas and Peucker, 1973) with a maximum camber threshold of $1 \mathrm{~m}$.

Calculation of additional metadata per reach: The final network consists of individual stream sections (source-to-node, node-to-node, node-to-outlet) which can further be partitioned into homogeneous reaches separated, e.g., by cataracts, bridges or gradient discontinuities. For each reach a set of additional parameters are derived and can be stored as metadata in the GIS data model. The features of interest are: length of reach, mean surface slope, winding, stream ordering number and corresponding river ID.

\section{Results AND Discussion}

Figure 4 shows the results for study area Fragant (Carinthia). As can be seen, the entire watershed was partitioned into ten sub-catchments for which the river network was derived as described in Sections 2.2 and 2.3. The individual catchment results were merged to a single network and Horton stream ordering numbers indicated by the different colors of the segments were calculated and assigned. Compared to the existing channel network (black), the results based on the high resolution ALS DTM are more detailed, more accurate, and the completeness is higher, all, w.r.t. the DTM shading as reference.

The benefits of the applied hierarchical approach emerge from Figure 5 featuring a rather flat part of the Hausruck (Upper Austria) dataset with a meandering river reach. Figure 5a was processed based on the raw DTM without prior visual examination and preprocessing. A series of bridges and other filtering artifacts are clearly visible and result in incorrect channel geometries. The errors are more serious for the reduced $5 \mathrm{~m}$ resolution (red) neglecting some relevant micro-topography features, but still unacceptable based on the high resolution $1 \mathrm{~m}$ grid (blue). The real channel courses, in turn, are revealed by the openness map (a) and the hill shading (b). The results shown in Figure 5b were obtained by removing the bridges from the DTM via manual editing and running the automatic preprocessing steps described in Section 2.2. Compared to (a) the results of (b) are improved with many of the artificial rivers (red) being successfully removed automatically based on the openness layer and only the relevant rivers 

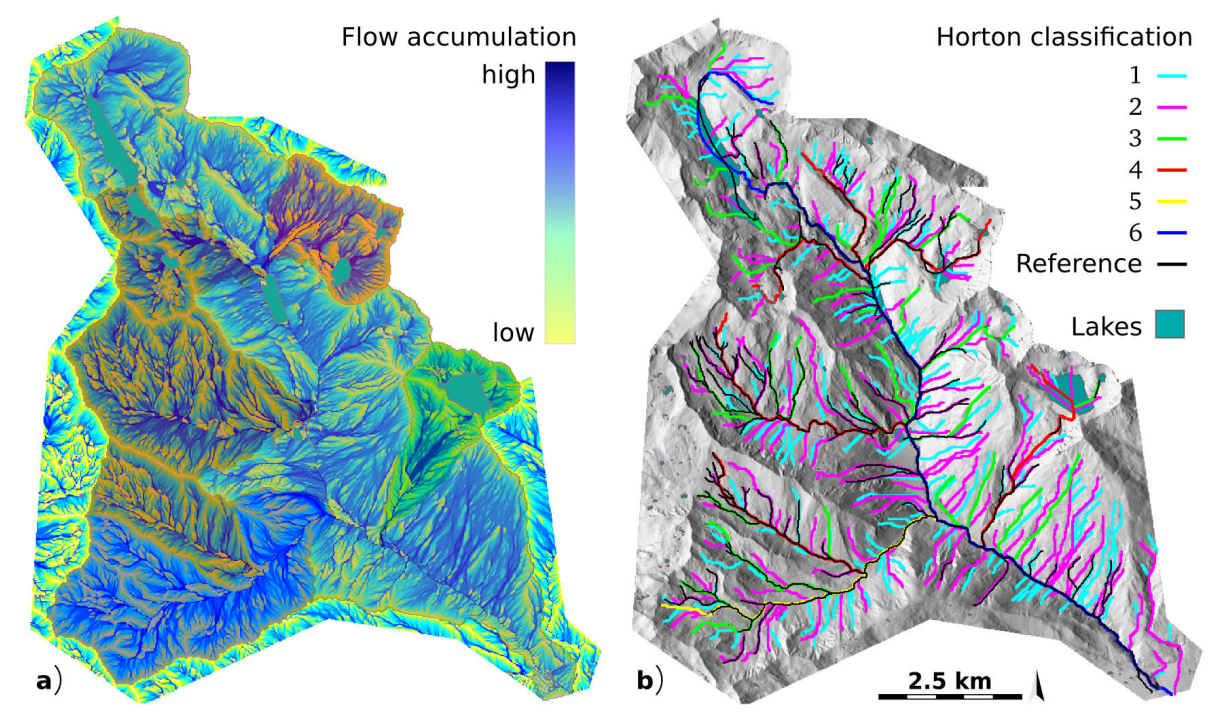

Figure 4: Results Carinthia, Fragant: (a) flow accumulation, sub-catchment areas marked in different color tones; (b) automatically derived river network (black=existing river network)

(blue) remaining. However, a closer look (c.f. detail of Figure 5b) indicates that still some of the derived channels get out of the creek bed and erroneously run through open fields. This is caused by remaining vegetation in the DTM due to incorrect filtering of the underlying ALS point cloud. As stated above, it is beyond the scope of this study to improve the geometric quality of the DTM. However, the results clearly show that correct river networks can only be obtained if the DTM strictly represents the water surface and is not affected by filtering errors. The effects are more serious in flat terrain (Hausruck) than in steep terrain (Fragant). A real improvement can only be achieved by stepping back to the ALS point cloud and by repeating the classification into ground and off-terrain points, at least in the vicinity of the rivers.

\section{Conclusions And OUtLook}

In this paper we presented an automatic workflow for deriving a topologically consistent, countrywide river network based on high resolution ALS DTM data. A hierarchical procedure consisting of (i) catchment delineation, (ii) DTM preprocessing, (iii) actual channel network derivation, and (iv) postprocessing
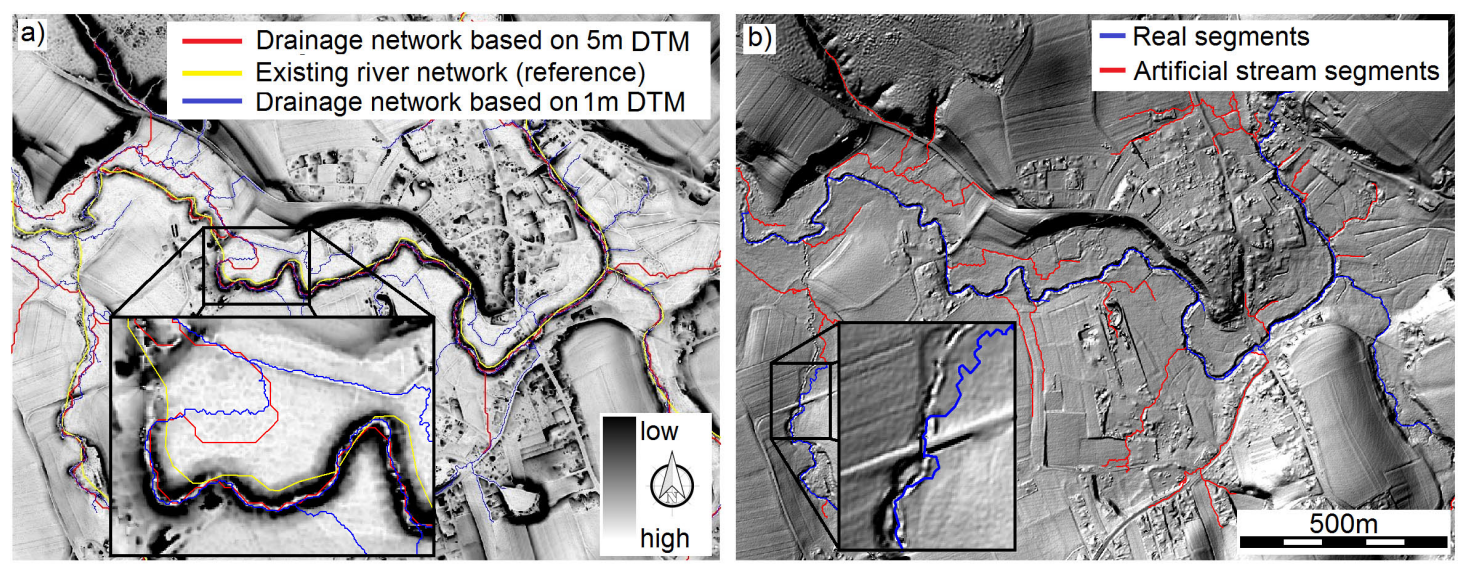

Figure 5: Detail of study area Upper Austria, Hausruck (Lon/Lat: 13 $\left.35^{\circ} 41^{\prime \prime} / 48^{\circ} 24^{\prime} 01^{\prime \prime}\right)$ : (a) openness map and channels derived from raw DTM (bridges not removed), (b) hill shading and channels derived from preprocessed DTM 
could be implemented using standard GIS software. Open source tools (GRASS GIS, SAGA) have proven to be equally suited as commercial products (ArcGIS). Additionally to using standard tools, embedding the channel network detection in a robust countrywide context, a procedure for adaptive smoothing of the DTM, and a method for classifying the raw results into real and artificial rivers are our genuine contributions. Subdivision into catchments of $10-20 \mathrm{~km}^{2}$, a hierarchical approach for the channel detection based on $5 \mathrm{~m}$ and $1 \mathrm{~m}$ DTM resolution and the automatic removal of artificial stream segments using an openness layer have been identified as key components for the derivation of a countrywide river network.

Satisfactory results w.r.t. completeness and accuracy could have been achieved for all terrain types (steep, undulating, flat) if the DTM correctly represented the water surface. However, problems occurred where the DTM contained bridges and river bank vegetation.

\section{ACKNOWLEDGMENTS}

This project was funded by the Austrian Federal Ministry of Agriculture, Forestry, Environment and Water Management (Project: BIMLFUW-UW.3.2.5/0040-VII/2010.) and partly supported by the FP7MC-IAPP project ChangeHabitat 2.

\section{REFERENCES}

Arge, L., J. Chase, P. Halpin, L. Toma, D. Urban, J. S. Vitter, and R. Wickremesinghe (2003). Flow Computation on Massive Grid Terrains. International Journal on Advances of Computer Science for Geographic Information Systems 7(4), 283-313.

Douglas, D. H. and T. K. Peucker (1973). Algorithms for the reduction of the number of points required to represent a digitized line or its caricature. The Canadian Cartographer 10(2), 112-122.

ESRI (Accessed: September 2011). Homepage of Environmental Systems Research Institute (ESRI). http://www.esri.com.

EU (2000). Water Framework Directive, 2000/60/EC. Official Journal of the European Union (OJL) 327, 173.

EU (2007). Infrastructure for Spatial Information in the European Community (INSPIRE), directive 2007/2/EC. Official Journal of the European Union (OJL) 50, 114.

Freeman, T. (1991). Calculating catchment area with divergent flow based on a regular grid. Computers and Geosciences 17(3), 413-422.

GRASS Development Team (Accessed: September 2011). Geographic Resources Analysis Support System (GRASS GIS) Software. USA: Open Source Geospatial Foundation. http://grass.osgeo.org.

Jasiewicz, J. and M. Metz (2011). A new GRASS GIS toolkit for Hortonian analysis of drainage networks. Computers and Geosciences 37(8), $1162-1173$.

Jenson, S. and J. O. Domingue (1988). Extracting topographic structure from digital elevation data for geographic information system analysis. Photogrammetric Engineering and Remote Sensing 54(11), 1593-1600.

Mandlburger, G., J. Otepka, W. Karel, W. Wagner, and N. Pfeifer (2009). Orientation and Processing of Airborne Laser Scanning Data (OPALS) - Concept and First Results of a Comprehensive ALS Software. In ISPRS Workshop Laserscanning 2009, Paris, France.

SAGA (Accessed: September 2011). Homepage of System for Automated Geoscientific Analyses (SAGA) Software. http://www.saga-gis.org.

Sofia, G., P. Tarolli, F. Cazorzi, and G. Dalla Fontana (2011). An objective approach for feature extraction: distribution analysis and statistical descriptors for scale choice and channel network identification. Hydrology and Earth System Sciences 15(5), 1387-1402.

Yokoyama, R., M. Shirasawa, and R. Pike (2002). Visualizing Topography by Openness: A New Application of Image Processing to Digital Elevation Models. Photogrammetric Engineering and Remote Sensing 68(3), 257266. 